\section{TATRA \\ MOUNTaiNS \\ Mathematical Publications}

DOI: $10.2478 / \mathrm{tmmp}-2021-0029$

Tatra Mt. Math. Publ. 80 (2021), 15-34

\title{
A QUINTIC SPLINE COLLOCATION METHOD FOR SOLVING TIME-DEPENDENT CONVECTION-DIFFUSION PROBLEMS
}

\author{
Abdelmajid El Hajaji ${ }^{1}$ - AbDelhafid Serghini ${ }^{2}-$ \\ Said Melliani ${ }^{3}$ - Jalila El GhordaF ${ }^{3}$ - Khalid Hilal ${ }^{3}$ \\ ${ }^{1}$ LESJEP Laboratory, FSJESJ, University Chouaïb Doukkali, El Jadida, \\ MOROCCO \\ ${ }^{2}$ ANAA Research Team, ESTO, LANO Laboratory, FSO, University Mohammed Premier, \\ Oujda, MOROCCO \\ ${ }^{3}$ LAMSC Laboratory, FST, University Sultan Moulay Slimane, Beni-Mellal, \\ MOROCCO
}

\begin{abstract}
In this paper, we develop a new numerical algorithm for solving a time dependent convection-diffusion equation with Dirichlet's type boundary conditions. The method comprises the horizontal method of lines for time integration and $\theta$-method, $\theta \in[1 / 2,1](\theta=1$ corresponds to the backward Euler method and $\theta=1 / 2$ corresponds to the Crank-Nicolson method) to discretize in temporal direction and the quintic spline collocation method. The convergence analysis of proposed method is discussed in detail, and it justified that the approximate solution converges to the exact solution of orders $O\left(\Delta t+h^{3}\right)$ for the backward Euler method and $O\left(\Delta t^{2}+h^{3}\right)$ for the Crank-Nicolson method, where $\Delta t$ and $h$ are mesh sizes in the time and space directions, respectively. It is also shown that the proposed method is unconditionally stable. This scheme is applied on some test examples, the numerical results illustrate the efficiency of the method and confirm the theoretical behaviour of the rates of convergence. Results shown by this method are in good agreement with the known exact solutions. The produced results are also more accurate than some available results given in the literature.
\end{abstract}

\section{Introduction}

The convection-diffusion equation is a parabolic partial differential equation combining the diffusion and the advection equation, which describes physical

(C) 2021 Mathematical Institute, Slovak Academy of Sciences.

2010 Mathematics Subject Classification: 41A15, 65K15, 90C56.

Keywords: convection-diffusion equation, $\theta$-method, quintic, spline collocation method, convergence.

() $(1) \Theta$ Licensed under the Creative Commons BY-NC-ND 4.0 International Public License. 


\section{A. EL HAJAJI-A. SERGHINI-S. MELLIANI- J. EL GHORDAF-K. HILAL}

phenomena where particles or energy or other physical quantities are transferred inside a physical system. For different problems, a convection-diffusion equation may be written in various forms. In order to confirm the theoretical results, many numerical methods have been developed (see, e.g., [13, 15, 23, 28, 31]). In 10,26, Natesan and Deb proposed higher-order numerical schemes for singularly perturbed parabolic reaction-diffusion problems. Kadalbajoo et al. in [25] used the B-spline collocation method on piecewise-uniform Shishkin mesh, which leads to a tridiagonal linear system. Surla et al. 32. consider the finite difference approximation of a singularly perturbed one-dimensional convection-diffusion two-point boundary value problem. The problem is numerically treated by a quadratic spline collocation method on a piecewise uniform slightly modified Shishkin mesh. Zhu et al. [36] introduce a coupled approach of local discontinuous Galerkin and standard finite element method for solving singularly perturbed convection-diffusion problems. A number of finite element methods have been developed for the simulation of convection-diffusion-reaction equations with small diffusion. One of the most popular ones is the Streamline Upwind Petrov-Galerkin (SUPG) method published in [2,17]. A completely different finite element approach for treating equations with small diffusion is the Finite Element Method Flux-Corrected Transport (FEMFCT) scheme [19,20].

Solution of a convection-diffusion-reaction equation in a time-dependent domain is highly demanded in many applications and have been examined by many authors (see, e.g. [16, 24, 27, 34]). In addition to a stabilized numerical method, an efficient approach is necessary to handle the mesh movement for a convection dominated convection-diffusion equation in time-dependent domains.

In [1, the authors approximate the equations of time-dependent convection diffusion problem, in space by a continuous finite element method with local projection stabilization and in time by a discontinuous Galerkin method. Xin Cai et al. [3] used the Finite Volume Method for time-dependent convection diffusion large Reynolds number problem, where two efficient numerical methods are constructed. One is Shishkin's finite volume method, the second one is Improved-Shishkin's finite volume method. J. Zhang and D. Yang [35] construct a parallel least-square finite element algorithm (PLS) for solving the first-order time-dependent convection-diffusion system. Clevero et al. [4,7,8] have developed a variety of parameter uniform convergent method based on Shishkin mesh.

The main objective of this study is to develop a user friendly, economical method which can work for time-dependent convection-diffusion equation by using a quintic splines collocation method and be compared with other numerical methods given in the literature, in particular with [12]. 


\section{A QUINTIC SPLINE COLLOCATION METHOD}

Consider the singularly perturbed initial-boundary value problem

$$
\left\{\begin{aligned}
\frac{\partial u}{\partial t}-L_{\epsilon} u=f, & (x, t) \in(0,1) \times(0, T], \\
u(x, 0)=\phi_{0}(x), & x \in[0,1], \\
u(0, t)=u(1, t)=0, & t \in] 0, T],
\end{aligned}\right.
$$

where $L_{\epsilon}: C(\bar{\Omega}) \cap C^{2,1}(\Omega) \rightarrow C(\Omega)$, with $\Omega=(0,1) \times(0, T]$, is defined by

$$
L_{\epsilon}:=\epsilon \frac{\partial^{2}}{\partial x^{2}}-a(x) \frac{\partial}{\partial x}-b(x, t) I, \quad(x, t) \in \Omega \times(0, T),
$$

with $a(x) \geq \widetilde{\alpha}>0, b=b(x, t) \geq 0$ on $\bar{\Omega}$. The diffusion coefficient $\epsilon$ is a small positive parameter, and $a, b, \phi_{0}$ are sufficiently smooth functions.

Here, we assume that the problem satisfies sufficient regularity and compatibility conditions which guarantee that the problem has a unique solution $u \in C(\bar{\Omega}) \bigcap C^{2,1}(\Omega)$ satisfying (see, [14, 21, 30])

$$
\left|\frac{\partial^{i+j} u(x, t)}{\partial r^{i} \partial t^{j}}\right| \leq k\left(1+\epsilon^{-i} e^{-\alpha(1-x) / \epsilon}\right) \text { on } \bar{\Omega} ; \quad 0 \leq j \leq 3 \quad \text { and } \quad 0 \leq i+j \leq 4,
$$

where $k$ is a constant.

In this paper, we will use the quintic B-spline to solve a time-dependent convection-diffusion problem (11). The main purpose is to analyse the efficiency of the quintic spline collocation method for such problems with sufficient accuracy. The procedure consists of a quintic spline approximation for the space derivative and a difference approximation for the time derivative. Such a combination of finite difference and quintic spline function techniques provide better accuracy than the finite difference methods. Therefore, the time derivative is approximated by horizontal method of line finite-difference representation and the space derivatives by quintic spline. In comparison with the existing well-known methods, our method is simple and numerically stable with the high accuracy approximation order. Numerical computations show that our results are well accepted.

The rest of this paper is organized as follows. In Section 2, we describe the continuous problem, its reduced problem and the classical bounds on the solution. In Section 3, we describe the approximation of the time derivative by means of the $\theta$-finite difference method, and the error in the temporal direction has been shown to be of one and second order. Also, we prove the stability of temporal discretization in this section. In Section 4, the formulation of the numerical method based on quintic spline functions is given. We give the convergence and error analysis of our method in Section 5. Finally, Section 6 describes the numerical experiments to corroborate the results predicted by the theory. 


\section{A. EL HAJAJI-A. SERGHINI-S. MELLIANI- J. EL GHORDAF-K. HILAL}

\section{The time semidiscretization and description of the $\theta$-method}

The observation period has to be specified first and it is set from today (time point $t=0$ ) to the time point $T$. This period will be divided into $M$ equally spaced time intervals with length $\Delta t=\frac{T}{M}$, and we denote it by $t_{m}=m \Delta t$, $m=0,1,2, \ldots, M$.

We discretize the variable time in (11) by means of $\theta$-method, $\theta \in\left[\frac{1}{2}, 1\right]$. Then the semi-discretization yields the following equation:

$$
\frac{u^{m+1}-u^{m}}{\triangle t}-\theta L_{\epsilon}^{m} u^{m+1}-(1-\theta) L_{\epsilon}^{m} u^{m}=f^{m+\theta},
$$

where $L_{\epsilon}^{m}: C\left(\bar{\Omega}_{x}\right) \bigcap C^{2}\left(\Omega_{x}\right) \rightarrow C\left(\Omega_{x}\right)$ is the differential operator defined by

$$
L_{\epsilon}^{m}=\epsilon \frac{\partial^{2}}{\partial x^{2}}+\beta^{m+\theta} \frac{\partial}{\partial x}+\gamma^{m+\theta} I
$$

with $u^{m}$ approximate the exact solution $u\left(x, t_{m}\right)$ at the time level

$$
t_{m}=m \Delta t, \quad \beta^{m+\theta}=-a(x) \quad \text { and } \quad \gamma^{m+\theta}=-b\left(x, t_{m}+\theta \Delta t\right) .
$$

From Equation (4), we have

$$
u^{m+1}-u^{m}-\triangle t\left(\theta L_{\epsilon}^{m} u^{m+1}-(1-\theta) L_{\epsilon}^{m} u^{m}\right)=\triangle t f^{m+\theta},
$$

and then

$$
\left(I-\theta \triangle t L_{\epsilon}^{m}\right) u^{m+1}=\left(I+(1-\theta) \triangle t L_{\epsilon}^{m}\right) u^{m}+\triangle t f^{m+\theta} .
$$

The choice of $\theta=1 / 2$ corresponds to the Crank-Nicolson method, and the choice of $\theta=1$ corresponds to the backward Euler method.

Then, the approximate problem of (11) is

$$
\left\{\begin{aligned}
p^{m+\theta} u_{x x}^{m+1}+q^{m+\theta}(x) u_{x}^{m+1}+l^{m+\theta}(x) u^{m+1}=g^{m+\theta}, & x \in \Omega_{x}, \\
u^{0}(x)=\phi_{0}(x), & x \in \Omega_{x}, \\
u^{m+1}(0)=u^{m+1}(1)=0, & 0 \leq m<M,
\end{aligned}\right.
$$

where for any $m \geq 0$ and for any $x \in \Omega_{x}=(0,1)$, we have:

$$
\begin{aligned}
p^{m+\theta} & =\theta \Delta t \epsilon, \\
q^{m+\theta}(x) & =\theta \Delta t \beta^{m+\theta}(x), \\
l^{m+\theta}(x) & =\theta \Delta t \gamma^{m+\theta}(x)-1, \\
g^{m+\theta}(x) & =-\left(I+(1-\theta) \Delta t L_{x}^{m}\right) u^{m}-\Delta t f^{m+\theta} .
\end{aligned}
$$

We have $u^{0}(x)=\phi_{0}(x)$. Then, for $0 \leq m \leq M-1, u^{m}$ being known, we obtain $u^{m+1}$ as a solution of the problem (6). The following theorem proves the order of convergence of the solution $u^{m}$ to $u\left(x, t_{m}\right)$. 


\section{A QUINTIC SPLINE COLLOCATION METHOD}

Theorem 2.1 (see [11, 18]). Problem (6) is second order convergent for $\theta=\frac{1}{2}$ and first order convergent for $\left.\theta \in] \frac{1}{2}, 1\right]$, i.e., there exists a constant Cte such that

$$
\begin{aligned}
& \left\|u\left(x, t_{m}\right)-u^{m}\right\|_{\infty} \leq \operatorname{Cte}(\triangle t)^{2} \quad \text { for } \theta=\frac{1}{2} \\
& \left.\left.\left\|u\left(x, t_{m}\right)-u^{m}\right\|_{\infty} \leq \operatorname{Cte} \triangle t \quad \text { for } \theta \in\right] \frac{1}{2}, 1\right] .
\end{aligned}
$$

For any $m \geq 0$, problem (6) has a unique solution and can be written in the following form

$$
\left\{\begin{aligned}
p u^{\prime \prime}(x)+q(x) u^{\prime}(x)+l(x) u(x) & =g(x), \quad x \in \Omega_{x} \\
u(0)=u(1) & =0 .
\end{aligned}\right.
$$

In the sequel of this paper, we will focus on the solution of problem (7).

\section{Spatial discretization and quintic spline collocation method}

In this section, we construct a quintic spline which approximates the solution $u$ of problem (7) in the interval $\Omega_{x}=(0,1) \subset \mathbb{R}$.

We denote by $\|$. $\|$ the Euclidean norm on $\mathbb{R}^{n+1},\|\cdot\|_{\infty}$ the uniform norm.

The method in this paper employs B-spline collocation for discretization, and it may be useful at this point to present a brief introduction to B-spline theory. B-splines are functions designed to generalize polynomials for the purpose of approximating arbitrary functions. One becomes familiar with them by understanding their qualitative behaviour and how to use them. If

$$
\prod:\left\{x_{-5}=\cdots=x_{-1}=x_{0}=0<x_{1}<x_{2}<\cdots<x_{n-1}<x_{n}=\cdots=x_{n+5}=1\right\}
$$

is a partition of $[0,1]$ and $h=\frac{1}{n}$ is the norm of the partition $\prod$.

Let $Q_{i}$ be quintic B-splines with knots at the points $x_{i}, i=0,1, \ldots, n$. The set of splines $\left\{Q_{-5}, Q_{-4}, \ldots, Q_{n-2}, Q_{n-1}\right\}$ forms a basis for functions defined over $[0,1]$. Thus, an approximation $u(x)$ to the exact solution $U^{q}(x)$ can be expressed in terms of the quintic B-splines as trial functions

$$
U^{q}(x)=\sum_{i=-5}^{n-1} \kappa_{i} Q_{i}(x),
$$

where $\kappa_{i}$ are time dependent quantities to be determined from boundary conditions and collocation form of the differential equations. 


\section{A. EL HAJAJI-A. SERGHINI-S. MELLIANI- J. EL GHORDAF-K. HILAL}

Each basis function $Q_{j}(x)$ is twice continuously differentiable.

By using the approximation (7), quintic B-splines, the nodal value $u$ and its first, second and third derivatives with respect to variable $x, U_{x}^{q}, U_{x x}^{q}, U_{x x x}^{q}$ at the nodes $x_{i}$.

By using the boundary conditions of problem (77) we obtain $\kappa_{-5}=U^{q}(0)=0$, and $\kappa_{n-1}=U^{q}(1)=0$.

In order to uniquely determine $\kappa_{-4}$ and $\kappa_{n-2}$, two linearly independent equations are required. By differentiating Eq. (77) with respect to variable $x \in[0,1]$, we have $\kappa_{-4}, \kappa_{n-2} \in \mathbb{R}$ (see APPENDIX), so

$$
U^{q}(x)=\mu(x)+S(x), \quad \text { with } \quad S=\sum_{i=-3}^{n-3} \kappa_{i} \bar{Q}_{i} \quad(\text { see APPENDIX). }
$$

According to 9], for any $U \in C^{10}([0,1])$

$$
\left\|D^{r}(U-u)\right\|_{\infty}=O\left(h^{6-r}\right) \quad \text { for } \quad r=0,1,2,3,
$$

where $D^{r}$ is the differential operator of order $r$ with respect to variable $x$.

We force the function $U^{q}(x)$ to satisfy the problem (7) at the nodal points of the partition $\Pi$. Thus, we have

$$
p \cdot S^{(2)}\left(x_{j}\right)+q\left(x_{j}\right) S^{(1)}\left(x_{j}\right)+l\left(x_{j}\right) S^{(0)}\left(x_{j}\right)=f\left(x_{j}\right)+O\left(h^{3}\right), \quad j=0, \ldots, n,
$$

where

$$
\begin{aligned}
f\left(x_{j}\right) & =g\left(x_{j}\right)-\lambda\left(x_{j}\right), \\
\lambda\left(x_{j}\right) & =p \cdot \mu^{(2)}\left(x_{j}\right)+q\left(x_{j}\right) \mu^{(1)}\left(x_{j}\right)+l\left(x_{j}\right) \mu^{(0)}\left(x_{j}\right), \quad j=0, \ldots, n .
\end{aligned}
$$

The quintic spline collocation method, that we present in this paper, constructs numerically a quintic spline $\widetilde{S}=\sum_{i=-5}^{n-1} \widetilde{\kappa}_{i} \bar{Q}_{i}$ which satisfies the equation of problem (77) at the points $x_{j}, j=0, \ldots, n$. It is easy to see that $\widetilde{\kappa}_{-5}=0, \widetilde{\kappa}_{-4} \in \mathbb{R}$, $\widetilde{\kappa}_{n-2} \in \mathbb{R}$ and $\widetilde{\kappa}_{n-1}=0$, and the coefficients $\widetilde{\kappa}_{i}, i=-3, \ldots, n-3$ satisfy the following

$$
p . \widetilde{S}^{(2)}\left(x_{j}\right)+q\left(x_{j}\right) \widetilde{S}^{(1)}\left(x_{j}\right)+l\left(x_{j}\right) \widetilde{S}^{(0)}\left(x_{j}\right)=f\left(x_{j}\right), \quad j=0, \ldots, n .
$$

Taking $C=\left[\kappa_{-3}, \ldots, \kappa_{n-3}\right]^{T}$ and $\widetilde{C}=\left[\widetilde{\kappa}_{-3}, \ldots, \widetilde{\kappa}_{n-3}\right]^{T}$, and using equations (10) and (11), we get:

$$
\begin{aligned}
& \left(P A_{h}^{(2)}+Q A_{h}^{(1)}+L A_{h}^{(0)}\right) C=F+E, \\
& \left(P A_{h}^{(2)}+Q A_{h}^{(1)}+L A_{h}^{(0)}\right) \widetilde{C}=F
\end{aligned}
$$




\section{A QUINTIC SPLINE COLLOCATION METHOD}

with:

$$
\begin{aligned}
F & =\left[f_{1}, \ldots, f_{n+1}\right]^{T}, \quad \text { and } \quad f_{j}=f\left(x_{i}\right), \\
E & =\left[O\left(h^{3}\right), \ldots, O\left(h^{3}\right)\right]^{T} \in \mathbb{R}^{n+1} \text { be the truncation error vector, } \\
P & =\left(\operatorname{diag}\left(\triangle t \theta \epsilon I_{j}\right)\right)_{0 \leq j \leq n}, \\
Q & =\left(\operatorname{diag}\left(\triangle t \theta \beta^{m+\theta}\left(x_{j}\right)\right)\right)_{0 \leq j \leq n}, \\
L & =\left(\operatorname{diag}\left(\triangle t \theta \gamma^{m+\theta}\left(x_{j}\right)-1\right)\right)_{0 \leq j \leq n}, \\
A_{h}^{(k)} & =\left(Q_{l}^{(k)}\left(x_{j}\right)\right)_{0 \leq j, l \leq n}, \quad k=0,1,2 .
\end{aligned}
$$

It is well-known that $A_{h}^{(k)}=\frac{1}{h^{k}} A_{k}$ for $k=0,1,2$ where matrices $A_{0}, A_{1}$ and $A_{2}$ are independent of $h$, with the matrix $A_{2}$ independent of $h$ given as follows 22

$$
A_{2}=\left[\begin{array}{cccccccc}
14 & 33 & 12 & 1 & 0 & 0 & \ldots & 0 \\
-4 & -12 & -4 & 0 & 0 & 0 & \ldots & 0 \\
\frac{1}{6} & \frac{1}{3} & -1 & \frac{1}{3} & \frac{1}{6} & 0 & \ldots & 0 \\
\vdots & \ddots & \ddots & \ddots & \ddots & \ddots & \ddots & \vdots \\
0 & \ldots & 0 & \frac{1}{6} & \frac{1}{3} & -1 & \frac{1}{3} & \frac{1}{6} \\
0 & \ldots & 0 & 0 & 0 & -4 & -12 & -4 \\
0 & \ldots & 0 & 0 & -1 & -12 & -33 & -14
\end{array}\right] .
$$

The results of this work are basically based on the invertibility of the matrix $A_{2}$. Then, in order to prove that $A_{2}$ is invertible we give the flowing lemma.

Lemma 3.1 (see [9]). Let $S \in \mathbb{S}_{k+1}$ such that $S=0$ on $\left[x_{p-1}, x_{p}\right] \cup\left[x_{q} \cup x_{q+1}\right]$, where $p<q$. If $s$ admits $r$ zeros in $\left[x_{p}, x_{q}\right]$, then $r \leq p-q-(k+1)$.

Theorem 3.2. The matrix $A_{2}$ is invertible.

P r o o f. Let $D=\left[d_{0}, \ldots, d_{n}\right]^{T}$ be a vector of $\mathbb{R}^{d+1}$ such that $A D=0$. If we put $S=\sum_{i=-3}^{n-3} d_{i+3} Q_{i}$, then we have $S$ is completely null outside the interval $[0,1]$,

$$
S(0)=S(1)=0 \quad \text { and } \quad S^{\prime \prime}\left(x_{i}\right)=0 \text { for any } i=0, \ldots, n \text {. }
$$


Since $S \in \mathbb{S}_{6}(I, \Pi)$, then $S^{\prime \prime} \in \mathbb{S}_{4}(I, \Pi)$. If we assume that $S^{\prime \prime} \neq 0$ in $\left[x_{0}, x_{n}\right]$, then using the above lemma and the fact that $S^{\prime \prime}$ has $n+1$ zeros in $\left[x_{0}, x_{n}\right]$, we conclude that $n+1 \leq n-4$, which is impossible. Therefore, $S^{\prime \prime}(x)=0$ for each $x \in[0,1]$. This means that the function $S$ is a piecewise linear polynomial in $[0,1]$. Since $S(0)=S(1)=0$, we obtain $S(x)=0$ for any $x \in[0,1]$. Consequently, $D=0$ and the matrix $A_{2}$ is invertible.

We deduce that (12) and (13) can be written also in the following form

$$
\begin{aligned}
P A_{2}\left(I+A_{2}^{-1} P^{-1}\left(h Q A_{1}+h^{2} L A_{0}\right)\right) C & =h^{2} F+h^{2} E, \\
P A_{2}\left(I+A_{2}^{-1} P^{-1}\left(h Q A_{1}+h^{2} L A_{0}\right)\right) \widetilde{C} & =h^{2} F .
\end{aligned}
$$

\subsection{Convergence analysis}

In order to determine the bounded of $\|C-\widetilde{C}\|$, we need the following remark.

Remark 1. For a small real $h$ such that

$$
\left\|A_{2}^{-1} P^{-1}\right\|_{\infty}\left(h\|Q\|_{\infty}\left\|A_{1}\right\|_{\infty}+h^{2}\|L\|_{\infty}\left\|A_{0}\right\|_{\infty}\right)<1
$$

the matrix $\left(I+A_{2}^{-1} P^{-1}\left(h Q A_{1}+h^{2} L A_{0}\right)\right)^{-1}$ exists, and

$$
\begin{aligned}
\|\left(I+A_{2}^{-1} P^{-1}\left(h Q A_{1}+h^{2} L A_{0}\right)\right)^{-1} & \|_{\infty}< \\
& \frac{1}{1-\left(h\|Q\|_{\infty}\left\|A_{1}\right\|_{\infty}+h^{2}\|L\|_{\infty}\left\|A_{0}\right\|_{\infty}\right)} .
\end{aligned}
$$

Hence, in this case, there exists a unique quintic spline that approximates the exact solution $u$ of problem (7).

Proposition 3.3. If we choose the real h such that

$$
\left\|A_{2}^{-1} P^{-1}\right\|_{\infty}\left(h\|Q\|_{\infty}\left\|A_{1}\right\|_{\infty}+h^{2}\|L\|_{\infty}\left\|A_{0}\right\|_{\infty}\right) \leq \frac{1}{2},
$$

then there exists a constant cte that depends only on the functions $p, q, l$ and $f$ such that

$$
\|C-\widetilde{C}\| \leq \mathrm{cte} \cdot h^{3}
$$

Pr o of. From relations (14) and (15) we have

$$
C-\widetilde{C}=h^{2}\left(P A_{2}\right)^{-1}\left(I+\left(P A_{2}\right)^{-1}\left(h Q A_{1}+h^{2} L A_{0}\right)\right)^{-1} E .
$$

Since $E=O\left(h^{3}\right)$, then there exists a constant $K_{1}$ such that $\|E\| \leq K_{1} h^{3}$. This implies that

$$
\|C-\widetilde{C}\| \leq K_{1} \frac{h^{2}\left\|\left(A_{2} P\right)^{-1}\right\|_{\infty}}{1-h^{2}\left\|\left(A_{2} P\right)^{-1}\right\|_{\infty}\left(h^{-1}\|Q\|_{\infty}\left\|A_{1}\right\|_{\infty}+\|L\|_{\infty}\left\|A_{0}\right\|_{\infty}\right)} h^{3} .
$$


Using the inequality

$$
\left\|A_{2}^{-1} P^{-1}\right\|_{\infty}\left(h\|Q\|_{\infty}\left\|A_{1}\right\|_{\infty}+h^{2}\|L\|_{\infty}\left\|A_{0}\right\|_{\infty}\right) \leq \frac{1}{2}, \quad \text { and } \quad 0<h \leq 1,
$$

we deduce that

$$
\|C-\widetilde{C}\| \leq K_{1} \frac{h^{2}\left\|\left(A_{2} P\right)^{-1}\right\|_{\infty}}{\left.\|Q\|_{\infty}\left\|A_{1}\right\|_{\infty}+\|L\|_{\infty}\left\|A_{0}\right\|_{\infty}\right)} h^{3} .
$$

Finally, we deduce that

$$
\|C-\widetilde{C}\| \leq \mathrm{cte} \cdot h^{3}
$$

Now, we are in position to prove the main theorem of our work.

THEOREM 3.4. The spline approximation $\widetilde{U}^{q}$ converges to the exact solution $u$ of problem (77) with order three, i.e., $\left\|u-\widetilde{U}^{q}\right\|_{\infty}=O\left(h^{3}\right)$.

P r o o f. It is well-known that $\left\|u-U^{q}\right\|_{\infty}=O\left(h^{3}\right)$ (see [9]), so $\left\|u-U^{q}\right\|_{\infty} \leq K h^{3}$, where $K$ is a positive constant. On the other hand, we have

$$
U^{q}(x)-\widetilde{U}^{q}(x)=\sum_{i=-2}^{n+2}\left(\kappa_{i}-\widetilde{\kappa}_{i}\right) Q_{i}(x) .
$$

Therefore, by using (17) and $\sum_{i=-2}^{n+2} Q_{i}(x) \leq 1$, we get

$$
\left|U^{q}(x)-\widetilde{U}^{q}(x)\right| \leq\|C-\widetilde{C}\| \sum_{i=-2}^{n+2} Q_{i}(x) \leq\|C-\widetilde{C}\| \leq \text { cte } \cdot h^{3} .
$$

Since $\left\|u-\widetilde{U}^{q}\right\|_{\infty} \leq\left\|u-U^{q}\right\|_{\infty}+\left\|U^{q}-\widetilde{U}^{q}\right\|_{\infty}$, we deduce the stated result.

TheOREm 3.5. We suppose that $u(x, t)$ is the solution of (11) and $u_{q}(x, t)$ is the approximate solution by our presented method, then we have,

$$
\begin{aligned}
\left\|u\left(x, t_{m}\right)-u_{q}\left(x, t_{m}\right)\right\|_{\infty} \leq C_{1}\left(\triangle t^{2}+h^{3}\right) & \text { for } \quad \theta=\frac{1}{2}, \\
\left\|u\left(x, t_{m}\right)-u_{q}\left(x, t_{m}\right)\right\|_{\infty} \leq C_{2}\left(\triangle t+h^{3}\right) & \text { for } \left.\quad \theta \in] \frac{1}{2}, 1\right],
\end{aligned}
$$

where $C_{1}$ and $C_{2}$ are finite constants. Therefore for sufficiently small $\triangle t$ and $h$, the solution of presented scheme (12) converges to the solution of initial boundary value problem (11) in the discrete $L_{\infty}-$ norm and the rates of convergence are

$$
O\left(\triangle t+h^{3}\right) \quad \text { and } O\left(\triangle t^{2}+h^{3}\right) .
$$




\subsection{Stability analysis}

We will now show that the fully discretized collocation cubic spline method is stable. The collocation procedure for solving boundary value problem (7) given by (15), i.e.,

where

$$
A \widetilde{C}=G,
$$

$$
\begin{gathered}
A=P A_{2}(I+U+V) \text { and } U=h A_{2}^{-1} P^{-1} Q A_{1}, \\
V=h^{2} A_{2}^{-1} P^{-1} L A_{0} \quad \text { and } \quad G=h^{2} F
\end{gathered}
$$

is said to be stable if for a perturbation of the data, i.e.,

$$
(A+\Gamma) \widetilde{\widetilde{C}}=G+\delta \text { and } \widetilde{\widetilde{U^{q}}}\left(x_{i}\right)=\widetilde{U^{q}}\left(x_{i}\right)+\delta_{i} \quad \text { pour } i=0, \ldots, n,
$$

there exists positive constants $k_{1}, k_{2}$ and $k_{3}$ independent of $n$ and $G$ in $\bar{\Omega}_{x}$, such that for all sufficiently large $n$

$$
(A+\Gamma) \widetilde{\widetilde{C}}=G+\delta
$$

has a unique solution for

where

$$
\|\Gamma\|_{\infty} \leq k_{1} \quad \text { and } \quad\left\|\widetilde{\widetilde{U}} q-\widetilde{U^{q}}\right\|_{\infty} \leq k_{2}\|\Gamma\|_{\infty}+k_{3}\|\widetilde{\delta}\|_{\infty}
$$

$$
\delta=\left(\delta_{0}, \delta_{2}, \ldots, \delta_{n}\right) \text { and } \widetilde{\delta}=\left(\widetilde{\delta_{0}}, \widetilde{\delta_{1}}, \ldots, \widetilde{\delta_{n}}\right)
$$

The functions $\widetilde{U^{q}}$ and $\widetilde{U^{q}}$ are uniquely determined by $\widetilde{\kappa}_{j}$ and $\widetilde{\widetilde{\kappa}}_{j},-5 \leq j \leq n-1$ in (15) and (18), respectively.

TheOREm 3.6. The collocation procedure (15) for solving the boundary value problem (7) is stable.

P r o of. As we know that

$\left\|A^{-1}\right\|_{\infty} \leq\left\|P^{-1}\right\|_{\infty}\left\|A_{2}^{-1}\right\|_{\infty}\left\|(I+U+V)^{-1}\right\|_{\infty} \leq \frac{\rho}{1-\|U+V\|_{\infty}}=k$, for $n \geq n_{0}$,

where $n_{0}$ is a sufficiently large positive real number and $k$ is a constant. Choose a positive constant $k_{1}<\frac{1}{2 k}$. Then whenever

exists because

$$
\|\Gamma\|_{\infty} \leq k_{1}, \quad(A+\Gamma)^{-1}=\left(I+A^{-1} \Gamma\right)^{-1} A^{-1}
$$

$\left\|A^{-1} \Gamma\right\|_{\infty} \leq\left\|A^{-1}\right\|_{\infty}\|\Gamma\|_{\infty}<\frac{1}{2}$, and in fact, $\left\|(A+\Gamma)^{-1}\right\|_{\infty} \leq 2 k$, for $n \geq n_{0}$.

After subtracting (15) from (18), we get

implicate that

$$
(A+\Gamma) \widetilde{\widetilde{C}}-A \widetilde{C}-\Gamma \widetilde{C}=\delta-\Gamma \widetilde{C},
$$

$$
\|\widetilde{\widetilde{C}}-\widetilde{C}\|_{\infty} \leq 2 k\left(\|\delta\|_{\infty}+\|\Gamma\|_{\infty}\|\widetilde{C}\|_{\infty}\right) .
$$




\section{A QUINTIC SPLINE COLLOCATION METHOD}

As it is assumed that $\alpha, \beta, \gamma, \phi_{0}$, and $\phi_{R}$ are sufficiently smooth on $\bar{\Omega}$ and

$$
\left|\frac{\partial^{i} u(x, t)}{\partial x^{i}}\right| \leq k \text { on } \bar{\Omega} \text { for } 0 \leq i \leq 4,
$$

so $\alpha^{m+\theta}, \beta^{m+\theta}, \gamma^{m+\theta}, \phi_{0}^{m+\theta}$, and $\phi_{R}^{m+\theta}$ will be sufficiently smooth on $\bar{\Omega}$ and

$$
\left|\frac{\partial^{i} u^{m+1}}{\partial x^{i}}\right| \leq k \quad \text { on } \bar{\Omega} \quad \text { for } \quad 0 \leq i \leq 4, \quad \text { and } \quad m=0,1, \ldots, M-1 .
$$

Further, since

$$
u\left(x_{i}\right)=U^{q}\left(x_{i}\right) \text { for all } i=0, \ldots, n
$$

and the inverse of the collocation matrix is bounded, i.e.,

$$
\left\|A^{-1}\right\|_{\infty} \leq k \quad \text { and } \quad\|\widetilde{C}\|_{\infty} \leq r, \quad \text { with } \quad r>0
$$

So

$$
\|\widetilde{\widetilde{C}}-\widetilde{C}\|_{\infty} \leq 2 k\left(\|\delta\|_{\infty}+r\|\Gamma\|_{\infty}\right)
$$

Now, since (19) therefore with the help of relation

$$
\widetilde{\widetilde{U^{q}}}\left(x_{i}\right)=\widetilde{U^{q}}\left(x_{i}\right)+\delta_{i} \quad \text { for } \quad i=0, \ldots, n,
$$

we have

$$
\begin{aligned}
& \max _{-2 \leq j \leq n-2}\left|\widetilde{\widetilde{c_{j}}}-\widetilde{c_{j}}\right| \leq 2 k r\|\Gamma\|_{\infty}+2 k\|\delta\|_{\infty}, \\
& \max _{-5 \leq j \leq n-1}\left|\widetilde{\widetilde{\kappa_{j}}}-\widetilde{\kappa_{j}}\right| \leq 2 k k^{\prime} r\|\Gamma\|_{\infty}+2 k k^{\prime}\|\delta\|_{\infty} .
\end{aligned}
$$

Further,

So

$$
\widetilde{\widetilde{U^{q}}}(x)-\widetilde{U^{q}}(x)=\sum_{-5 \leq j \leq n-1}\left(\widetilde{\tilde{\kappa}_{j}}-\widetilde{\kappa_{j}}\right) B_{j}(x)
$$

$$
\left|\widetilde{\widetilde{U^{q}}}\left(x_{i}\right)-\widetilde{U^{q}}\left(x_{i}\right)\right| \leq \max _{-5 \leq j \leq n-1}\left|\widetilde{\widetilde{\kappa}_{j}}-\widetilde{\kappa_{j}}\right| \sum_{-5 \leq j \leq n-1}\left|B_{j}\left(x_{i}\right)\right|, \text { for } i=0, \ldots, n,
$$

and like

therefore

$$
\sum_{-5 \leq j \leq n-1}\left|B_{j}\left(x_{i}\right)\right| \leq 1
$$

$$
\left\|\widetilde{\widetilde{U^{q}}}-\widetilde{U^{q}}\right\|_{\infty} \leq k_{2}\|\Gamma\|_{\infty}+k_{3}\|\widetilde{\delta}\|_{\infty}
$$

where $k_{2}=r k k^{\prime}$ and $k_{3}=2 k k^{\prime}$. 


\section{A. EL HAJAJI-A. SERGHINI-S. MELLIANI- J. EL GHORDAF-K. HILAL}

\section{Numerical experiments}

In this section, we verify experimentally theoretical results obtained in the previous section. If the exact solution is known, then at time $t \leq T$ the maximum error $E_{\epsilon}^{\max }$ can be calculated as:

$$
E_{\epsilon}^{\max }=\max _{x \in[0,1], t \in[0, T]}\left|u_{q}^{M, N}(x, t)-u(x, t)\right| .
$$

Otherwise, it can be estimated by the following double mesh principle:

$$
E_{\epsilon, M, N}^{\max }=\max _{x \in[0,1], t \in[0, T]}\left|u_{q}^{M, N}(x, t)-u_{q}^{2 M, 2 N}(x, t)\right|,
$$

where $u_{q}^{M, N}(x, t)$ is the numerical solution on the $M+1$ grids in space and $N+1$ grids in time, and $u_{q}^{2 M, 2 N}(x, t)$ is the numerical solution on the $2 M+1$ grids in space and $2 N+1$ grids in time.

In this section, we present the numerical results of present method on two problems presented in the paper of the authors A.ElHajaji et al. [12]. We tested the accuracy of this method for different values of $N, M, \epsilon$ and compared the obtained results to the ones given in [12].

\subsection{Example 1.}

Consider the time-dependent convection-diffusion problem (11) with the following data:

$$
\Omega=(0,1) \times(0,1),
$$

and

$$
\begin{aligned}
\phi_{0} & =0, \\
a(x) & =1+x^{2}+\frac{1}{2} \sin (\pi x), \\
b(x, t) & =1+x^{2}+\sin \left(\frac{\pi t}{2}\right), \\
f(x, t) & =x^{3}(1-x)^{3}+t(1-t) \sin (\pi t) .
\end{aligned}
$$

Table 1 shows values of the maximum error (max_error) obtained in our numerical experiments and the one obtained in [12]. Table 1] illustrates the comparative performance of our method with another existing method [12. The obtained approximate numerical solutions show that our proposed method maintains better accuracy compared with a recent other method [12] based on the quintic spline collocation method.

From these tables, we can say that the results of our schemes are acceptable, and conclude that the proposed schemes are feasible and valid. We observe that present method is nearly of three order convergence with respect to these error norms. 


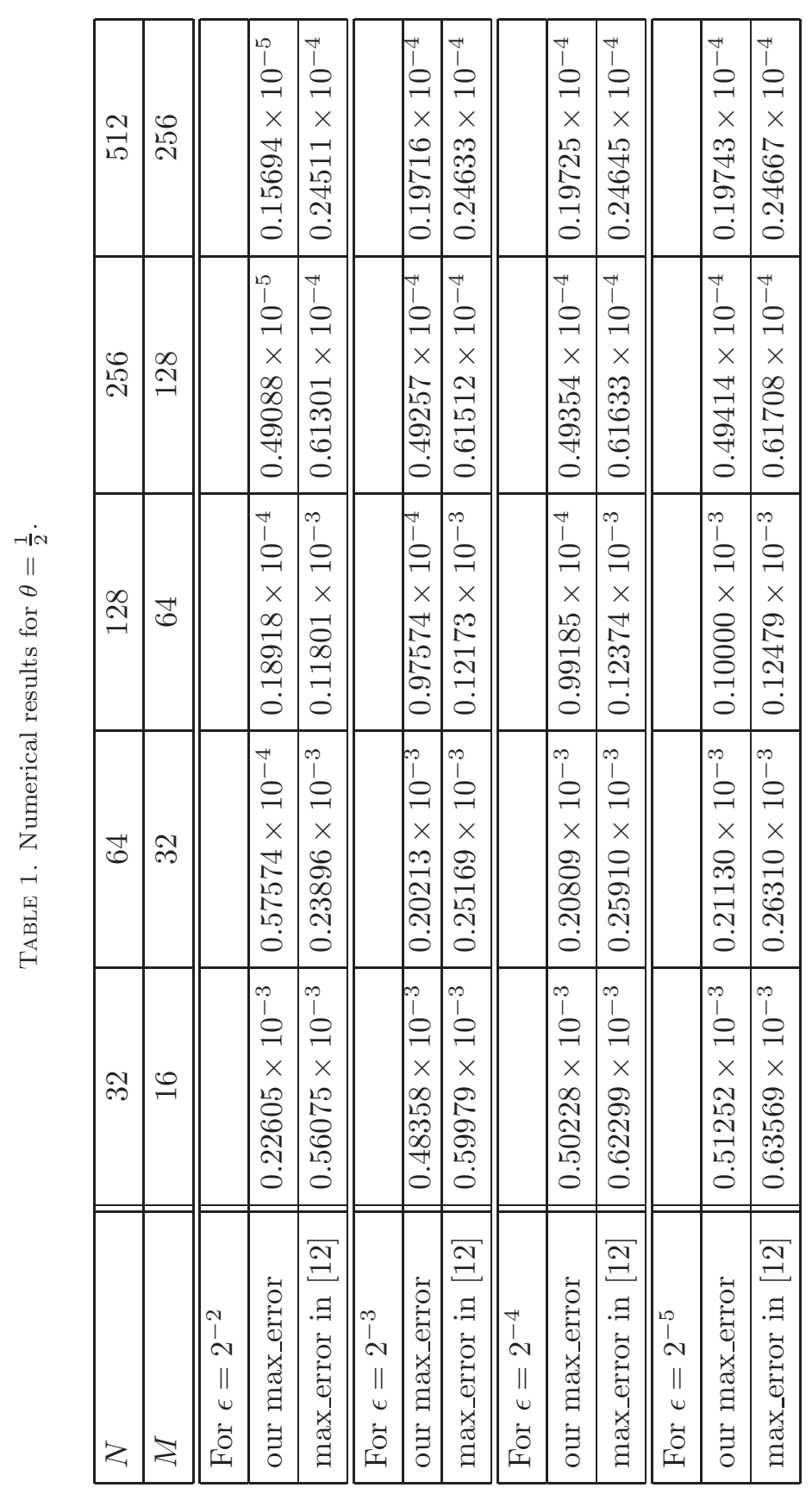


A. EL HAJAJI-A. SERGHINI-S. MELLIANI- J. EL GHORDAF-K. HILAL

\subsection{Example 2 .}

Consider the time-dependent convection-diffusion problem (11) with the following data:

$$
\Omega=(0,1) \times(0,1),
$$

and

$$
\begin{aligned}
\phi_{0} & =0, \\
a(x) & =4+4 x-x^{2}-e^{x}, \\
b(x, t) & =5 x t, \\
f(x, t) & =3 \sin (\pi x)+5\left(e^{t}-1\right) .
\end{aligned}
$$

Table 2 shows values of the maximum error (max_error) obtained in our numerical experiments and the one obtained in 6, 12]. Table 2 illustrates the comparative performance of our method with another existing method [6, 12]. The obtained approximate numerical solutions show that our proposed method maintains better accuracy compared with a recent other method 6, 12 based on the quintic spline collocation method.

From these tables, we can say that the results of our schemes are acceptable, and conclude that the proposed schemes are feasible and valid. We observe that present method is nearly of three order convergence with respect to these error norms.

\section{Conclusion}

The numerical method is developed to solve a time-dependent convectiondiffusion problems. This method is based on $\theta$-method for the temporal discretization and the quintic spline collocation method in the spatial direction. We have shown the convergence of the method and provided that the parameters $\epsilon, h$ and $\Delta t$ satisfy the relation (16). Moreover, we have provided an error estimate of order $O\left(\Delta t^{2}+h^{3}\right)$ with respect to the maximum norm: $\|$. $\|_{\infty}$. Numerical experiments were performed on two known models to validate the convergence and efficiency of the method. Comparisons of the computed results with exact solutions showed that the scheme is capable of solving the timedependent convection-diffusion equation and is also capable of producing highly accurate solutions with minimal computational effort for both time and space. The computational results show that the proposed numerical method is an efficient alternative method to the one proposed in [6, 12]. 


\begin{tabular}{|c|c|c|c|c|c|c|c|c|c|c|c|c|c|c|c|c|c|c|c|c|c|}
\hline$\frac{N}{i n}$ & $\begin{array}{l}0 \\
\stackrel{2}{N} \\
\stackrel{2}{2}\end{array}$ & & \begin{tabular}{|l}
$* *$ \\
$*$ \\
$*$ \\
$*$
\end{tabular} & \begin{tabular}{|l}
$* *$ \\
$*$ \\
$*$ \\
$*$
\end{tabular} & $\begin{array}{l}* * \\
* \\
* \\
*\end{array}$ & & $\mid$\begin{tabular}{c|}
0 \\
1 \\
0 \\
0 \\
$\times$ \\
$\times$ \\
$\infty$ \\
0 \\
0 \\
0 \\
0 \\
0 \\
0
\end{tabular} & $\mid$\begin{tabular}{|c|} 
\\
1 \\
0 \\
0 \\
$x$ \\
$x$ \\
0 \\
0 \\
0 \\
$\sim$ \\
0 \\
0 \\
\end{tabular} & 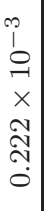 & & $\begin{array}{l}20 \\
1 \\
0 \\
-1 \\
\times \\
20 \\
0 \\
0 \\
0 \\
0 \\
0\end{array}$ & 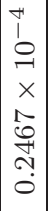 & 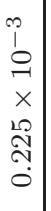 & & $\begin{array}{l}20 \\
1 \\
0 \\
-1 \\
\times \\
0 \\
0 \\
0 \\
0 \\
0 \\
0\end{array}$ & 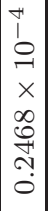 & 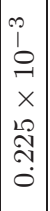 & & $\left|\begin{array}{l}20 \\
1 \\
0 \\
0 \\
\times \\
\times \\
0 \\
0 \\
0 \\
0 \\
0 \\
0\end{array}\right|$ & 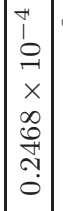 & 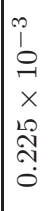 \\
\hline $\begin{array}{l}\stackrel{0}{\mathscr{D}} \\
\stackrel{2}{*}\end{array}$ & 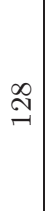 & & & $\begin{array}{l}* \\
* \\
* \\
* \\
*\end{array}$ & $\begin{array}{l}* * \\
* \\
* \\
*\end{array}$ & & 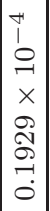 & 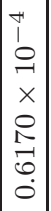 & $\begin{array}{l}0 \\
1 \\
0 \\
-1 \\
\times \\
+ \\
\stackrel{5}{9} \\
\stackrel{0}{0}\end{array}$ & & 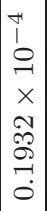 & 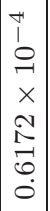 & $\begin{array}{l}\infty \\
i \\
0 \\
- \\
\times \\
o \\
\stackrel{9}{+} \\
\dot{0}\end{array}$ & & 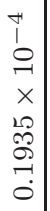 & $\begin{array}{l}+1 \\
1 \\
0 \\
-1 \\
x \\
0 \\
i \\
-1 \\
\dot{0} \\
0\end{array}$ & 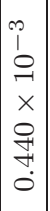 & & 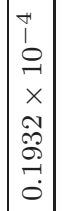 & $\begin{array}{l}+1 \\
1 \\
0 \\
0 \\
x \\
x \\
0 \\
1 \\
\overrightarrow{0} \\
\dot{0}\end{array}$ & $\begin{array}{l}0 \\
1 \\
0 \\
0 \\
x \\
0 \\
+4 \\
\vdots \\
0\end{array}$ \\
\hline$\underset{\stackrel{\infty}{\sim}}{\sim} \mid$ & రే & & \begin{tabular}{|l}
$* *$ \\
$*$ \\
$*$ \\
$*$
\end{tabular} & \begin{tabular}{|l}
$*$ \\
$*$ \\
$*$ \\
$*$ \\
$*$
\end{tabular} & $\begin{array}{l}* * \\
* \\
* \\
* \\
*\end{array}$ & & 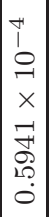 & 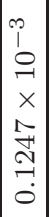 & $\begin{array}{l}\infty \\
0 \\
1 \\
0 \\
\qquad \\
\times \\
0 \\
\stackrel{1}{0} \\
0 \\
0\end{array}$ & & 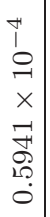 & 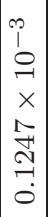 & 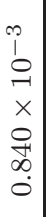 & & 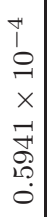 & 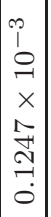 & 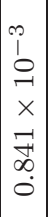 & & $\mid \begin{array}{l}1 \\
1 \\
1 \\
0 \\
-1 \\
x \\
7 \\
7 \\
0 \\
0 \\
0 \\
0\end{array}$ & 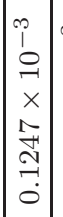 & $\begin{array}{l}\infty \\
1 \\
0 \\
1 \\
x \\
-7 \\
0 \\
0 \\
0\end{array}$ \\
\hline ठే & ๙ิ & & 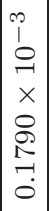 & 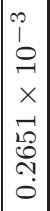 & 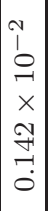 & & 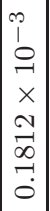 & 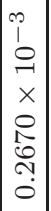 & 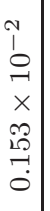 & & \begin{tabular}{l|}
0 \\
1 \\
0 \\
-1 \\
$\times$ \\
10 \\
$\overrightarrow{0}$ \\
-1 \\
$\overrightarrow{0}$
\end{tabular} & 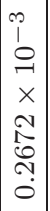 & 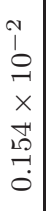 & & 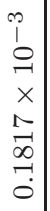 & 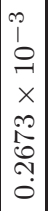 & 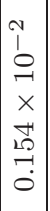 & & 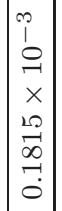 & 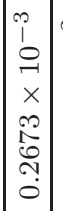 & $\begin{array}{l}1 \\
1 \\
0 \\
0 \\
x \\
10 \\
10 \\
-1 \\
0 \\
0\end{array}$ \\
\hline ஸै & $\stackrel{0}{-1}$ & & 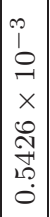 & 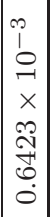 & 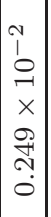 & & 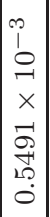 & $\begin{array}{l}\infty \\
1 \\
0 \\
0 \\
x \\
0 \\
0 \\
\infty \\
0 \\
0 \\
0 \\
0\end{array}$ & 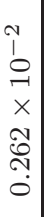 & & $\begin{array}{l}0 \\
1 \\
0 \\
0 \\
0 \\
x \\
0 \\
0 \\
0 \\
10 \\
0 \\
0\end{array}$ & 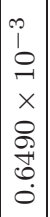 & $\begin{array}{l}1 \\
1 \\
0 \\
0 \\
\times \\
b \\
b \\
\stackrel{2}{0} \\
0\end{array}$ & & $\begin{array}{l}0 \\
i \\
0 \\
0 \\
\cdots \\
x \\
0 \\
0 \\
0 \\
20 \\
0 \\
0\end{array}$ & 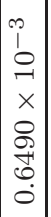 & 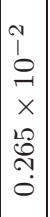 & & $\begin{array}{l}0 \\
1 \\
1 \\
0 \\
-1 \\
x \\
0 \\
0 \\
10 \\
10 \\
0 \\
0\end{array}$ & $\mid$\begin{tabular}{l|}
0 \\
1 \\
0 \\
0 \\
$\times$ \\
$\times$ \\
$-\overrightarrow{9}$ \\
$\overrightarrow{+}$ \\
0 \\
0
\end{tabular} & 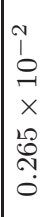 \\
\hline z & $z$ & $\begin{array}{l}1 \\
1 \\
N \\
\| \\
w \\
w \\
\tilde{0} \\
0\end{array}$ & 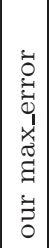 & 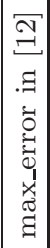 & 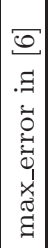 & $\mid \begin{array}{l}0 \\
1 \\
v \\
11 \\
u \\
u \\
\tilde{0} \\
0\end{array}$ & 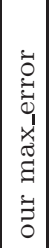 & 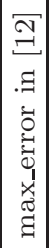 & 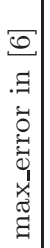 & 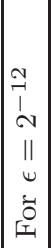 & 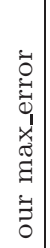 & 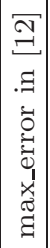 & 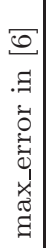 & 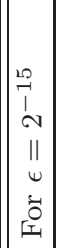 & 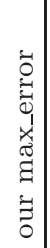 & 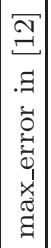 & 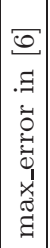 & 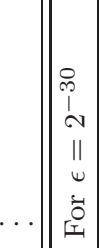 & 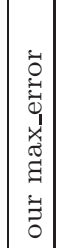 & 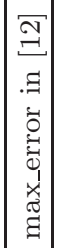 & 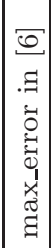 \\
\hline
\end{tabular}




\section{APPENDIX}

In this appendix, we will first derive the leading behaviour for differentiating Eq. (77) with respect to variable $x \in[0,1]$, we get

$$
p U_{x x x}^{q}(x)+q(x) U_{x x}^{q}(x)+\left(q^{\prime}(x)+l(x)\right) U_{x}^{q}(x)+l^{\prime}(x) U^{q}(x)=g^{\prime}(x) .
$$

By taking $x=x_{j}$, with $j \in\{0, n\}$ and putting the values of

$$
Q_{m}(x), \quad Q_{m}^{\prime}(x), \quad Q_{m}^{\prime \prime}(x) \text { and } Q_{m}^{\prime \prime \prime}(x),
$$

we have

$$
\frac{p}{2 h^{2}} \kappa_{j}^{(4)}+\frac{q_{j}}{6 h} \kappa_{j}^{(5)}+\frac{q_{j}^{\prime}+l_{j}}{120} \kappa_{j}^{(6)}+\frac{h l_{j}^{\prime}}{120} \kappa_{j}^{(7)}=h g_{j},
$$

where

$$
\begin{aligned}
& \kappa_{j}^{(4)}=\left(-\kappa_{j-2}+2 \kappa_{j-1}-2 \kappa_{j+1}+\kappa_{j+2}\right), \\
& \kappa_{j}^{(5)}=\left(\kappa_{j-2}+2 \kappa_{j-1}-6 \kappa_{j}+2 \kappa_{j+1}+\kappa_{j+2}\right), \\
& \kappa_{j}^{(6)}=\left(-\kappa_{j-2}-10 \kappa_{j-1}+10 \kappa_{j+1}+\kappa_{j+2}\right), \\
& \kappa_{j}^{(7)}=\left(\kappa_{-2}+26 \kappa_{j-1}+66 \kappa_{j}+26 \kappa_{j+1}+\kappa_{j+1}\right) .
\end{aligned}
$$

We solve the system (23), we get

$$
\begin{aligned}
\kappa_{-4} & =\frac{1}{b_{0}}\left(h g_{0}-c_{0} \kappa_{0}-d_{0} \kappa_{1}-e_{0} \kappa_{2}\right), \\
\kappa_{n-2} & =\frac{1}{d_{n}}\left(h g_{n}-a_{n} \kappa_{n-2}-b_{n} \kappa_{n-1}-c_{n} \kappa_{n}\right),
\end{aligned}
$$

where

$$
\begin{aligned}
& a_{n}=\frac{h l_{n}^{\prime}}{120}+\frac{q_{n}}{6 h}-\frac{p}{2 h^{2}}-\frac{q_{n}^{\prime}+l_{n}}{120}, \\
& b_{n}=\frac{13 h l_{n}^{\prime}}{60}+\frac{q_{n}}{3 h}+\frac{p}{h^{2}}-\frac{q_{n}^{\prime}+l_{n}}{12}, \\
& c_{n}=\frac{11 h l_{n}^{\prime}}{20}-\frac{q_{n}}{h}, \\
& d_{n}=\frac{13 h l_{n}^{\prime}}{60}+\frac{q_{n}}{3 h}+\frac{q_{n}^{\prime}+l_{n}}{12}-\frac{p}{h^{2}}, \\
& e_{n}=\frac{h l_{n}^{\prime}}{120}+\frac{q_{n}^{\prime}+l_{n}}{120}+\frac{q_{n}}{6 h}+\frac{p}{2 h^{2}} .
\end{aligned}
$$




\section{A QUINTIC SPLINE COLLOCATION METHOD}

So,

$$
U^{q}(x)=\mu(x)+S(x)
$$

with

$$
\mu(x)=\frac{g_{0}}{b_{0}} h Q_{-1}(x)+\frac{g_{n}}{b_{n}} h Q_{n+1}(x) \quad \text { and } \quad S=\sum_{i=-3}^{n-3} \kappa_{i} \bar{Q}_{i}
$$

with

$$
\begin{aligned}
& \bar{Q}_{0}=Q_{0}-\frac{c_{0}}{b_{0}} Q_{-1}, \\
& \bar{Q}_{1}=Q_{1}-\frac{d_{0}}{b_{0}} Q_{-1}, \\
& \bar{Q}_{2}=Q_{2}-\frac{e_{0}}{b_{0}} Q_{-1}, \\
& \bar{Q}_{3}=Q_{3}, \\
& \vdots \\
& \bar{Q}_{n-3}=Q_{n-3}, \\
& \bar{Q}_{n-2}=Q_{n-2}-\frac{a_{n}}{d_{n}} Q_{n+1}, \\
& \bar{Q}_{n-1}=Q_{n-1}-\frac{b_{n}}{d_{n}} Q_{n+1}, \\
& \bar{Q}_{n}=Q_{n}-\frac{c_{n}}{d_{n}} Q_{n+1} .
\end{aligned}
$$

\section{REFERENCES}

[1] AhMED, N.-MATTHIES, G.-TOBISKA, L.-XIE, H.: Discontinuous Galerkin time stepping with local projection stabilization for transient convection-diffusion-reaction problems, Comput. Methods Appl. Mech. Engrg. 200 (2011), no. 21-22, 1747-1756,

[2] BROOKS, A.N.-HUGHES, T. J.R.: Streamline upwind/Petrov-Galerkin formulations for convection dominated flows with particular emphasis on the incompressible NavierStokes equations, Comput. Methods Appl. Mech. Engrg. 32 (1982), 199-259.

[3] CAI, X.-CAI, D. - LU, M.: Finite volume method for time-dependent convection diffusion large Reynolds number problem. In: Second International Conference on Information and Computing Science, 2009, (ICIC '09), pp. 360-363, doi: 10.1109/ICIC.2009.296.

[4] CAI, X.-LIU, F.: A Reynolds uniform scheme for singularly perturbed parabolic differential equation, Anziam J. 47 (2005) (C), C 633-C 648. 


\section{A. EL HAJAJI-A. SERGHINI-S. MELLIANI- J. EL GHORDAF-K. HILAL}

[5] ClaVERO, C.-JORGE, J. C.-LISBONA, F.: Uniformly convergent scheme on a nonuniform mesh for convection-diffusion parabolic problems, J. Comput. Appl. Math. 154 (2003), 415-429.

[6] CLAVERO, C.-GRACIA, J. L.-STYNES, M.: , A simpler analysis of a hybrid numerical method for time-dependent convection-diffusion problems, J. Comput. Appl. Math. 235 (2011), 5240-5248.

[7] Clavero, C.-JORGE, J. C.-LiSBOnA, F.-ShishKin, G. I.: A fractional step method on a special mesh for the resolution of multidimensional evolutionary convectiondiffusion problems. Appl. Numer. Math. 27 (1998), no. 3, 211-231.

[8] CLAVERO, C.-JORGE, J. C.-LISBONA, F.: Uniformly convergent schemes for singular perturbation problems combining alternating directions and exponential fitting techniques. In: (Miller J. J.H. ed.) Applications of Advanced Computational Methods for Boundary and Interior Layers. Adv. Comput. Methods Bound. Inter. Layers, 2, Boole Press, Dublin, 1993, pp. 33-52.

[9] DE BOOR, C.: A Practical Guide to Splines. In: Applied Mathematical Sciences Vol. 27, Springer-Verlag, Berlin, 1978.

[10] DEB, R.-NATESAN, S.: Higher-order time accurate numerical methods for singularly perturbed parabolic partial differential equations, Int. J. Comput. Math. 86 (2009), no. 7, $1204-1214$.

[11] EL HAJAJI, A.-HILAL, K.-SERGHini, A.-MERMRI, EL BEKKEY, M.: Pricing American bond options using a cubic spline collocation method. Bol. Soc. Parana. Mat, 32 (2014) no. 2, 189-208.

[12] EL MERzGUiOUI, M.-EL HAJAJI, A.- HILAL, K.-CHADLI, L. S.: A numerical method for solving time-dependent convection-diffusion problems, Bol. Soc. Parana. Mat, (3) 35 (2017), no. 1, 217-228.

[13] JICHUN LI: Uniform convergence of discontinuous finite element methods for singularly perturbed reaction-diffusion problems, Comput. Math. Appl. 44 (2002), no. 1-2, 231-240.

[14] FRIEDMAN, A.: Partial Differential Equation of Parabolic Type. Robert E. Krieger Publishing Co., Huntington, NY, 1983.

[15] JICHUN LI-NAVON, I. M.: Uniformly convergent finite element methods for singularly perturbed elliptic boundary value problems: convection-diffusion type, Comput. Methods Appl. Mech. Engrg. 162 (1998), no. 1-4, 49-78.

[16] HINDMARSH, A. C.-GRESHO, P. M.-GRIFFITHS, D. F.: The stability of explicit Euler time-integration for certain finite difference approximations of the multi-dimensional advection-diffusion equation, Int. J. Numer. Meth. Fluids 4 (1984), 853-897.

[17] HUGHES, T. J. R.-BROOKS, A.: A multidimensional upwind scheme with no crosswind diffusion, (T. J. R. Hughes, ed.) In: Finite Element Methods for Convection Dominated Flows, (Papers, Winter Ann. Meeting Amer. Soc. Mech. Engrs., New York, 1979), AMD Vol. 34, Amer. Soc. Mech. Engrs. (ASME), New York, 1979, pp. 19-35.

[18] KADALBAJOO, M.K.-TRIPATHI, L.P.-KUMAR, A.: A cubic B-spline collocation for a numerical solution of generalized Black-Scholes equation, Math. Comput. Modelling 55 (2012), no. 3-4, 1483-1505. 


\section{A QUINTIC SPLINE COLLOCATION METHOD}

[19] KUZMIN, D.: Explicit and implicit FEM-FCT algorithms with flux linearization, J. Comput. Phys. 228 (2009), no. 7, 2517-2534.

[20] KUZMIN, D- MÖLLER, M.-TUREK, S.: High-resolution FEM-FCT schemes for multidimensional conservation laws, Comput. Methods Appl. Mech. Engrg. 193 (2004), 4915-4946.

[21] LAdYZEnSKAJA, O. A.-SOLONNIKOV, V. A.-URAL'CEVA, N. N.: Linear and Quasilinear Equations of Parabolic Type. In: Amer. Math. Soc. Transl. Vol. 23, Providence, RI. 1968.

[22] EL BEKKEY MERMRI.-SERGHINI, A.-EL HAJAJI, A.-HILAL, K. : A Cubic Spline Method for Solving a Unilateral Obstacle Problem, American J. Comput. Math. 2 (2012), no. 3, doi: 10.4236/ajcm.2012.23028.

[23] LI, JICHUN: High-order finite difference schemes for differential equations containing higher derivatives, Appl. Math. Comput. 171 (2005), no. 2, 1157-1176.

[24] MITCHELL, A. R.-GRIFFITHS, D. F.: Upwinding by Petrov-Galerkin methods in convection-diffusion problems, J. Comput. Appl. Math. 6 (1980), 219-228.

[25] KADALBAJOO, MOHAN, K.-YADAW, A.S.: B-Spline collocation method for a twoparameter singularly perturbed convection-diffusion boundary value problems, Appl. Math. and Comput. 201 (2008), no. 1-2, 504-513.

[26] NATESAN, S.-DEB, R.: A robust numerical scheme for singularly perturbed parabolic reaction diffusion problems, Neural Parallel Sci. Comput. 16 (2008), no. 3, 419-433.

[27] NG-STYNES, M. J.-O'RIORDAN, E.-STYNES, M.: Numerical methods for time-dependent convection-diffusion equations, J. Comput. Appl. Math. 21 (1988), no. 3, 289-310.

[28] LI, JICHUN: Convergence and superconvergence analysis of finite element methods on highly nonuniform anisotropic meshes for singularly perturbed reaction-diffusion problems, Appl. Numerical Math. 36 (2001), no. 2-3, 129-154.

[29] RAMOS, J. I.: (2005) An exponentially fitted method for singularly perturbed, one-dimensional, parabolic problems, Appl. Math. Comput. 161 (2005), 513-523.

[30] ROOS, H.-G.-STYNES, M.-TOBISKA, L.: Robust Numerical Methods for Singularly Perturbed Differential Equations Convection-diffusion-reaction and Flow Problems. (2nd edition). In: Springer Series in Computational Mathematics, Vol. 24, Springer-Verlag, Berlin, 2008.

[31] LI, JICHUN: Finite element analysis for a nonlinear diffusion model in image processing, Appl. Math. Lett. 15 (2002), no. 2, 197-202.

[32] SURLA, K.-TEOFANOV, L.-UZELAC, Z.: A robust layer-resolving spline collocation method for a convection-diffusion problem, Appl. Math. Comput. 208 (2009), no. 1, 76-89.

[33] SURLA, K.-JERKOVIĆ, V.: Some possibilities of applying spline collocations to singular perturbation problems. In: Numerical Methods and Approximation Theory, Vol. II, Univ. Novi Sad, Novi Sad, 1985. pp. 19-25.

[34] YU, C. C.-HEIMICH, J. C.: Petrov-Galerkin methods for the time-dependent convective transport equation, Internat. J. Numer. Methods Engrg. 23 (1986), 883-901. 


\section{A. EL HAJAJI-A. SERGHINI-S. MELLIANI- J. EL GHORDAF-K. HILAL}

[35] ZHANG, J.-YANG, D.: (2011) Parallel least-squares finite element method for time dependent convection-diffusion system, Computing 91 (2011), no. 3, 217-240.

[36] ZHU, P.-XIE, Z.-ZHOU, S.: A uniformly convergent continuous-discontinuous Galerkin method for singularly perturbed problems of convection-diffusion type, Appl. Math. Comput. 217 (2011), no. 9, 4781-4790.

Received March, 32020

\author{
Abdelmajid El Hajaji \\ Department of OEE \\ ENCGJ \\ University of Chouaib Doukkali \\ El Jadida \\ MOROCCO \\ E-mail: a_elhajaji@yahoo.fr \\ Abdelhafid Serghini \\ Department of Informatics \\ ESTO \\ Mohammed First University \\ 60050 Oujda \\ MOROCCO \\ E-mail: a.serghini@ump.ma \\ Said Melliani \\ Jalila El Ghordaf \\ Khalid Hilal \\ Department of Mathematics \\ FST \\ University Sultan Moulay Slimane \\ Beni-Mellal \\ MOROCCO \\ E-mail: saidmelliani@gmail.com \\ elg_jalila@yahoo.fr \\ hilal.khalid@yahoo.fr
}

